\title{
Concurrent Plantar Stress Sensing and Energy Harvesting Technique by Piezoelectric Insole Device and Rectifying Circuitry for Gait Monitoring in the Internet of Health Things
}

\author{
Shuaibo Kang ${ }^{1}$, Jingjing Lin ${ }^{1}$, Junliang Chen ${ }^{1}$, Yanning Dai ${ }^{1}$, Zhiheng Wang ${ }^{1}$ and Shuo Gao ${ }^{1,2, *}$ \\ ${ }^{1}$ School of Instrumentation Science and Optoelectronic Engineering, Beihang University, Beijing, China \\ ${ }^{2}$ Beijing Advanced Innovation Center for Big Data-Based Precision Medicine, Interdisciplinary Innovation Institute of Medicine \\ and Engineering, Beihang University, Beijing, China \\ *shuo_gao@buaa.edu.cn
}

\begin{abstract}
Concurrent high force detection accuracy and extended battery lifetime are strongly expected in wearable gait monitoring systems, which are important for many Internet of Health Things (IoHT) applications. In this article, a piezoelectric insole device and rectifying circuitry based technique is presented to achieve these two ultimate goals. Here, walking induced positive and negative charges are separated for plantar stress detection and energy harvesting respectively, realizing the two functions concurrently. Experimental results demonstrate that first, the high detection sensitivity of $55 \mathrm{mN}$ and responsivity of $231 \mathrm{mV} / \mathrm{N}$ are achieved, satisfying the need for diagnosing various diseases; second, energy of $1.6 \mathrm{pJ}$ is stored during a walking event, consequently extending the battery lifetime. The developed technique enhances the development of gait monitoring in IoHT.
\end{abstract}

Keywords-Gait monitoring; piezoelectric insole; plantar stress sensing; energy harvesting

\section{INTRODUCTION}

Gait analysis, based on plantar pressure detection, benefits varieties of applications, such as disease surveillance and analysis [1-2], health monitoring [3-5], shoe designing [6], and sports training [7]. By integrating it with the Internet of Health Things (IoHT) architecture, a wearable assistant is able to provide physical data for further processing and analysis [8-9]. Conventional techniques for gait analysis are piezoresistive, capacitive, piezoelectric based architectures, satisfying different utilization purposes [10-11]. However, for long-term monitoring applications (e.g., chronic disease monitoring), limited battery lifetime in conventional products brings users inconvenience [12-13]. To solve this issue, plenty of attempts have been made to boost the battery's capability [14]. Nevertheless, no commercial product on the market provides an ideal solution, giving rise to the increasing research interests in energy harvesting field. Among them, harvesting motion-induced mechanical energy shows the potential in extending the system's operation period in different application scenarios, hence triggering worldwide interests.

Recent literature [15-19] reports that some research groups have developed energy harvesting techniques to collect walking induced mechanical energy by using piezoelectric and triboelectric effects. However, those techniques lack force sensing function. Hence, the energy harvesting system has to be integrated into the insole plantar sensing system for gait monitoring purpose, causing the increased volume in system size, circuitry complexity and component cost, which are undesired for commercial products. In [20], a piezoelectric and rectifying circuitry based technique for concurrent force sensing and energy harvesting is presented for touch panels utilization. Nevertheless, only part of the touch induced positive and negative voltages are used to interpret the force amplitude, which will lower detection accuracy. Hence, concurrent high force detection accuracy and energy harvesting are highly expected for gait monitoring systems in IoHT scenarios.

In this work, a piezoelectric based gait monitoring and energy harvesting technique is presented. Here, motion-induced positive charges are used for plantar stress sensing, while their counterparts are harvested. The presented technique provides high force detection accuracy and extends battery lifetime simultaneously, while in a simple and low-cost manner, advancing the gait monitoring applications in IoHT.

\section{MethodolOGY}

\section{A. Sensor Fabrication}

To concurrently detect the plantar pressure and harvest energy, a multi-layer piezoelectric insole device is designed and fabricated, as shown in Fig. 1 (a). The polyvinylidene fluoride (PVDF) film $(52 \mu \mathrm{m})$, which is placed in the middle as force sensing layer, generates charges when force is applied. The top

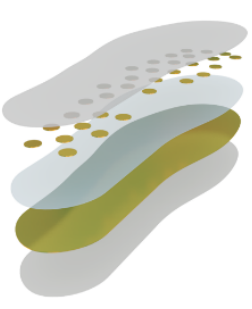

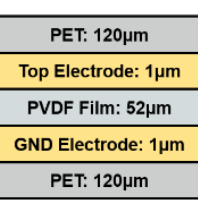

(a)

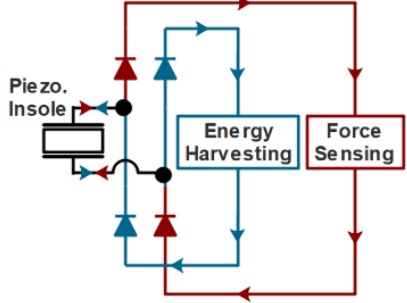

(b)
Fig. 1 (a) Architecture of the piezoelectric insole device. (b) Bridge rectifier based circuit for concurrent force sensing and energy harvesting. 


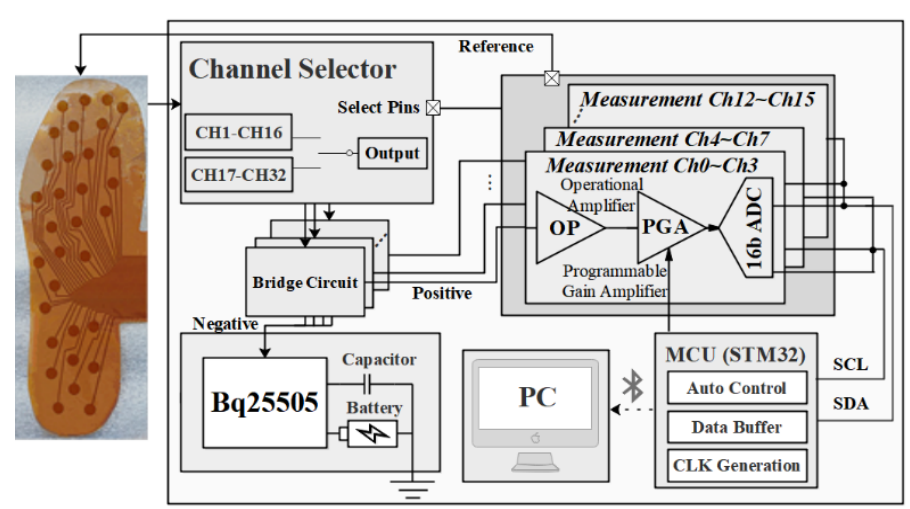

(a)

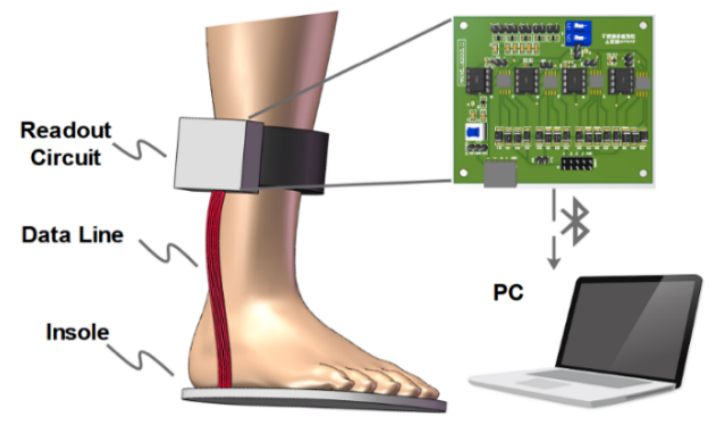

(c)

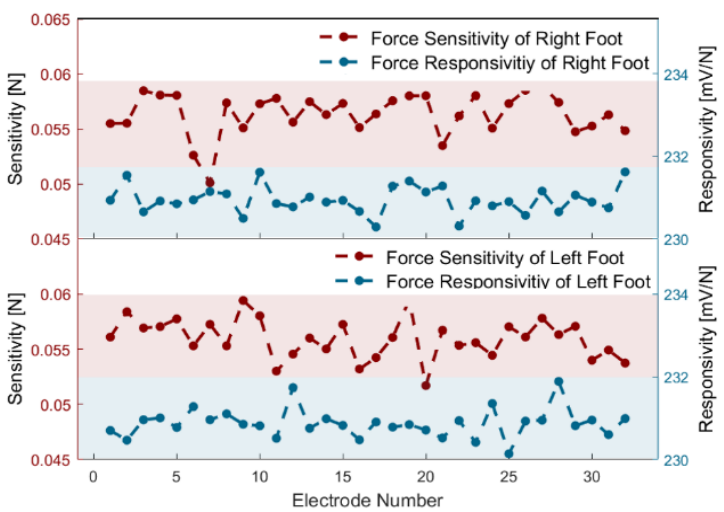

(b)

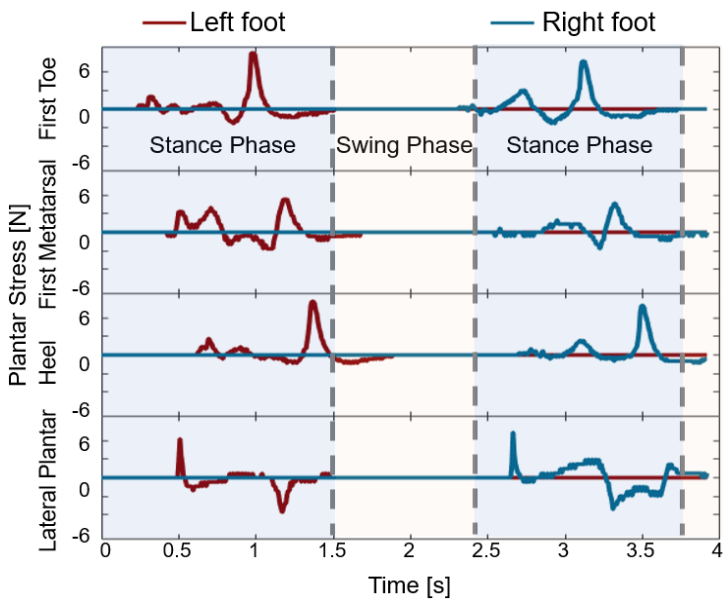

(d)

Fig. 2 (a) Block diagram of the readout circuit. (b) Sensitivity and responsivity of the fabricated insole force sensing device. (c) An overview of the gait monitoring system. (d) Gait monitoring during walking event.

and ground electrode layers $(1 \mu \mathrm{m})$ which conduct induced charges coat the PVDF layer. The top electrodes of one insole device is made of 32 circular copper sheets. The 32 copper electrodes are distributed according to the suggestions in [21-22] for diverse diseases analysis. The ground electrode is a continuous copper sheet. The two outermost polyethylene terephthalate (PET) layers are the substrates $(100 \mu \mathrm{m})$ for the copper electrodes, protecting the $\mathrm{Cu} / \mathrm{PVDF} / \mathrm{Cu}$ sandwiched structure. Different layers are laminated together, forming the piezoelectric insole device.

\section{B. Circuitry Design}

In [23], we learn that with a piezoelectric insole, a complete walking event (step tread and release) results in positive and negative voltage components. In this work, the positive amplitude indicates the tread action, based on the direction of the dipole. According to human walking behavior, the velocity of contacting with the ground is normally faster than its counterpart [23]. Hence, the absolute amplitude of stepping is higher compared to that of releasing. Considering that stepping tread related plantar stress is more important for disease analysis, e.g., diabetic foot diagnosis, the positive amplitudes are chosen for plantar stress sensing and the energy generated by release is harvested for extending battery's lifetime.

As shown in Fig. 1 (b), modifications on the classic rectifier bridge are made to separate the positive and negative signals, then conduct them into the force sensing as well as energy harvesting systems. When positive charges are generated, the current, flows through the red path and enters the force sensing system due to the one way conduction effect of diodes. The positive charges induced by the plantar pressure are conveyed to amplifiers. Then the amplified signals are sampled by the analog-to-digital converter (ADC) chip. Digital gait data are sent to microcontroller unit (MCU) through I2C protocol, and then transmitted to the host computer through Bluetooth module for further processing and analysis.

Relatively, during the release period, the generated negative charges flow through the blue path and are stored by the energy harvesting system, which is shown in the Fig. 1 (b). A passive, ultra-low current, cold-start energy management chip (Bq25505) is used to manage the energy usage for the entire system.

The entire circuit block diagram of concurrent gait monitoring and energy harvesting is shown in Fig. 2 (a). 


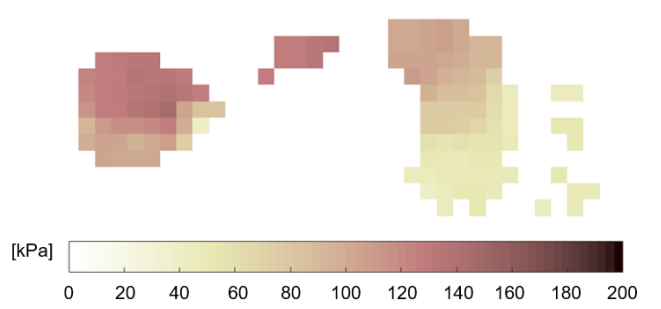

(a)

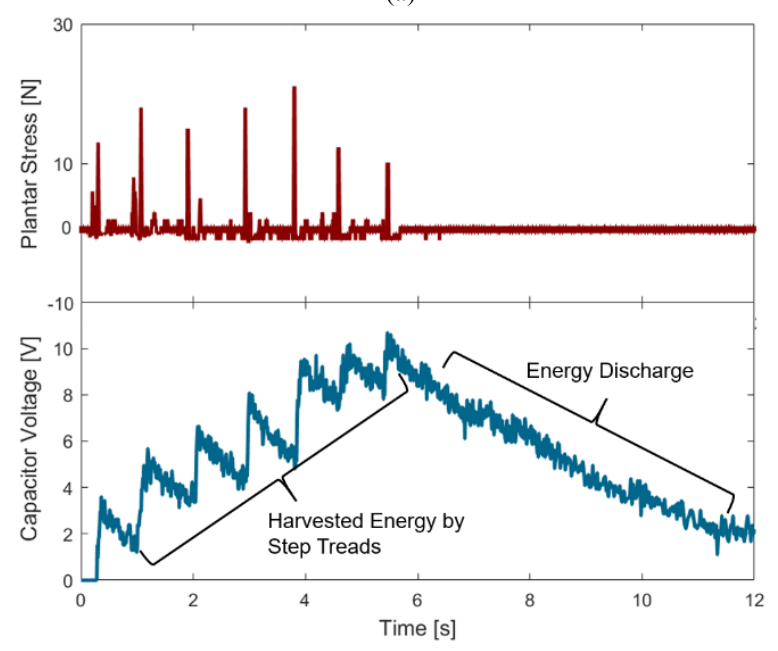

(b)

Fig. 3 (a) 2D stress distribution after the averaging based algorithm. (b) Experimental results of concurrent force sensing and energy harvesting.

TABLE I . SUMMARY OF THE MAJOR CiRCUIT PARAMETERS

\begin{tabular}{|c|c|}
\hline \multicolumn{2}{|c|}{ Circuit Parameters } \\
\hline Input Dynamic Range & $0 \mathrm{~V}$ to $+5 \mathrm{~V}$ \\
\hline ADC Resolution & $16 \mathrm{Bit}$ \\
\hline Data Rate & $230,400 \mathrm{bps}$ \\
\hline Scanning Rate & $150 \mathrm{~Hz}$ \\
\hline Processing Time & $8 \mathrm{~ms}$ \\
\hline
\end{tabular}

\section{RESULTS AND DISCUSSION}

\section{A. Device Performance}

Force detection sensitivities and responsivities of the fabricated insoles are examined by the Berlincourt method [24]. The corresponding results are presented in Fig. 2 (b). We can learn that the uniformity of both parameters is good, indicating the fabrication process is reliable. The average plantar stress detection sensitivity and responsivity are $55 \mathrm{mN}$ and $231 \mathrm{mV} / \mathrm{N}$, respectively. The results here demonstrate that the performance of the fabricated insoles can satisfy the need for diagnosing various diseases [25-26].

\section{B. Gait Monitoring}

An overview of the gait monitoring system is shown in Fig. 2 (c). The system is practically used by a subject to study its performance during walking is shown in Fig. 2 (d). Results of 4 main detection areas (first toe, heel, first metatarsal, and lateral plantar) for disease analysis are demonstrated, showing that a complete walking cycle can be monitored. The 2-dimensional plantar stress distribution of the right foot is presented in Fig. 3 (a), indicating that detailed plantar stress information can be captured. The system performance for gait monitoring is shown in Table I.

\section{Concurrent Force Sensing and Energy Harvesting}

To clearly demonstrate the performance of concurrent force sensing and energy harvesting, we showcase the result from one electrode in Fig. 3 (b). It can be observed that during one complete walking event, the stepping tread induced positive voltage components are interpreted as gait information. The negative component related electric energy is stored. Here we use a $1 \mathrm{nF}$ capacitor to clearly recognize the increment of harvested energy during walking. With the voltage across the capacitor, harvested energy can be calculated by Eq. 1 .

$$
E=\frac{1}{2} U^{2} C
$$

where $E$ represents the energy stored in the capacitor, $C$ is the capacitance of the capacitor and $U$ is the capacitor voltage. As shown in Fig. 3 (b), the average harvested energy for the 32 electrodes during a walking cycle is $1.6 \mathrm{pJ}$. The total stored energy is around $1.6 \mathrm{pJ}$ by the piezoelectric insoles during an entire walking cycle.

\section{CONCLUSION}

High plantar stress detection accuracy and long battery lifetime are two highly desired attributes for gait monitoring in the Internet of Health Things (IoHT) applications. The technique presented in this article satisfies these needs by implementing a piezoelectric insole device with rectifying circuitry. The amount of harvested energy is rather small during each walking cycle. But taking the numerous amount of everyday walking events and the potential prospect of higher mechanical-to-electrical conversion efficiency in advanced piezoelectric materials and low-power technique into consideration, the developed technique has a strong potential in providing sufficient power for future gait monitoring electronics.

\section{REFERENCES}

[1] H. Stolze et al., "Comparative analysis of the gait disorder of normal pressure hydrocephalus and Parkinson's disease," Neurol Neurosurg Psychiatry, vol. 70, no. 3, pp. 289-297, 2001.

[2] H. Stolze et al., "Gait analysis in idiopathic normal pressure hydrocephalus-which parameters respond to the CSF tap test?," Clinical Neurophysiology, vol. 111, no. 9, pp. 1678-1686, 2000.

[3] W. Tao, T. Liu, R. Zheng, and H. J. S. Feng, "Gait analysis using wearable sensors," Sensors, vol. 12, no. 2, pp. 2255-2283, 2012.

[4] J. Bae, K. Kong, N. Byl, and M. Tomizuka, "A mobile gait monitoring system for gait analysis," in 2009 IEEE International Conference on Rehabilitation Robotics, 2009, pp. 73-79: IEEE.

[5] I. González, J. Fontecha, R. Hervás, and J. Bravo, "An Ambulatory System for Gait Monitoring Based on Wireless Sensorized Insoles," Sensors, vol. 15, no. 7, pp. 16589-16613, 2015.

[6] M. Khoury, A. Wolf, E. M. Debbi, A. Herman, A. J. F. Haim, and a. international, "Foot center of pressure trajectory alteration by biomechanical manipulation of shoe design," Foot \& Ankle International, vol. 34, no. 4, pp. 593-598, 2013.

[7] J. A. García-Pérez, P. Pérez-Soriano, S. Llana, A. Martínez-Nova, D. J. G. Sánchez-Zuriaga, and posture, "Effect of overground vs treadmill running 
on plantar pressure: Influence of fatigue," Gait \& posture, vol. 38, no. 4 , pp. 929-933, 2013.

[8] P. S. Malvade, A. K. Joshi, and S. P. Madhe, "IoT based monitoring of foot pressure using FSR sensor," in 2017 International Conference on Communication and Signal Processing (ICCSP), 2017, pp. 0635-0639: IEEE.

[9] S. Jeon, C. Lee, Y. Han, D. Seo, and I. Jung, "The smart shoes providing the gait information on IoT," in 2017 IEEE International Conference on Consumer Electronics (ICCE), 2017, pp. 108-109: IEEE.

[10] R. Calio et al., "Piezoelectric energy harvesting solutions," Sensors (Basel), vol. 14, no. 3, pp. 4755-90, Mar 102014.

[11] L. Yiming et al., "Active Piezoelectric Energy Harvesting: General Principle and Experimental Demonstration," Journal of Intelligent Material Systems and Structures, vol. 20, no. 5, pp. 575-585, 2008.

[12] P. Kamalinejad, C. Mahapatra, Z. Sheng, S. Mirabbasi, V. C. Leung, and Y. L. J. I. C. M. Guan, "Wireless energy harvesting for the Internet of Things," IEEE Communications Magazine, vol. 53, no. 6, pp. 102-108, 2015.

[13] Y.-H. Lam, W.-H. Ki, C.-Y. J. I. T. o. C. Tsui, and S. I. E. Briefs, "Integrated low-loss CMOS active rectifier for wirelessly powered devices," IEEE Transactions on Circuits and Systems II: Express Briefs, vol. 53, no. 12, pp. 1378-1382, 2006.

[14] S. Priya, "Advances in energy harvesting using low profile piezoelectric transducers," Journal of Electroceramics, vol. 19, no. 1, pp. 167-184, 2007.

[15] J. Kymissis, C. Kendall, J. Paradiso, and N. Gershenfeld, "Parasitic power harvesting in shoes," in Digest of Papers. Second International Symposium on Wearable Computers (Cat. No. 98EX215), 1998, pp. 132139: IEEE.

[16] J. Zhao and Z. You, "A shoe-embedded piezoelectric energy harvester for wearable sensors," Sensors (Basel), vol. 14, no. 7, pp. 12497-510, Jul 11 2014.

[17] G. Zhu, P. Bai, J. Chen, and Z. Lin Wang, "Power-generating shoe insole based on triboelectric nanogenerators for self-powered consumer electronics," Nano Energy, vol. 2, no. 5, pp. 688-692, 2013.
[18] G. D. Szarka, B. H. Stark, and S. G. Burrow, "Review of Power Conditioning for Kinetic Energy Harvesting Systems," IEEE Transactions on Power Electronics, vol. 27, no. 2, pp. 803-815, 2012.

[19] S. S. Kumar and K. J. I. J. o. R. E. R. Kashwan, "Research study of energy harvesting in wireless sensor networks," International Journal of Renewable Energy Research, vol. 3, no. 3, pp. 745-753, 2013.

[20] S. Gao, C.-y. Huang, and L. Wu, "Piezoelectric material based technique for concurrent force sensing and energy harvesting for interactive displays," in 2017 IEEE SENSORS, 2018, pp. 1-3: IEEE.

[21] C. Lou et al., "Dynamic balance measurement and quantitative assessment using wearable plantar-pressure insoles in a pose-sensed virtual environment," Sensors, vol. 18, no. 12, p. 4193, 2018.

[22] S. Kärki, J. Lekkala, H. Kuokkanen, J. J. S. Halttunen, and A. A. Physical, "Development of a piezoelectric polymer film sensor for plantar normal and shear stress measurements," Elsevier, vol. 154, no. 1, pp. 57-64, 2009.

[23] A. Almusallam, R. Torah, D. Zhu, M. Tudor, and S. Beeby, "Screenprinted piezoelectric shoe-insole energy harvester using an improved flexible PZT-polymer composites," in Journal of Physics: Conference Series, 2013, vol. 476, no. 1, p. 012108: IOP Publishing.

[24] S. L. Kok, K. T. Lau, and Q. Ahsan, "Substrate-free thick-film lead zirconate titanate (pzt) performance measurement using berlincourt method," in Advanced Materials Research, 2014, vol. 895, pp. 204-210: Trans Tech Publ.

[25] A. Muro-De-La-Herran, B. Garcia-Zapirain, and A. J. S. Mendez-Zorrilla, "Gait analysis methods: An overview of wearable and non-wearable systems, highlighting clinical applications," Sensors, vol. 14, no. 2, pp. 3362-3394, 2014

[26] J. S. Petrofsky et al., "The effect of body fat, aging, and diabetes on vertical and shear pressure in and under a waist belt and its effect on skin blood flow," Sensors Diabetes Technology \& Therapeutics, vol. 12, no. 2, pp. 153-16 